www.jmscr.igmpublication.org

Impact Factor (SJIF): 6.379

Index Copernicus Value: 79.54

ISSN (e)-2347-176x ISSN (p) 2455-0450

crossrefDOI: https://dx.doi.org/10.18535/jmscr/v6i12.120

\title{
Urinary Candidiasis: Species Distribution and Anti-fungal susceptibility to Fluconazole and Amphotericin B.
}

\author{
Authors \\ Shashir Wasudeorao Wanjare ${ }^{1}$, Swati Dilip Gupta ${ }^{2 *}$, Preeti Rajeev Mehta ${ }^{3}$ \\ Department of Microbiology, Seth G.S. Medical College \& KEM Hospital, Mumbai \\ *Corresponding Author \\ Swati Dilip Gupta \\ Department of Microbiology, Seth G.S. Medical College \& KEM Hospital, Mumbai, India. \\ (M) 7387306968, Email: dr.swati29@gmail.com
}

\begin{abstract}
Introduction: Urinary candiasis is common in hospitalized patients. Candiduria may be a result of colonization or infection of urinary tract. The differentiation can be made on basis of presence of pyuria and significant urinary colony counts. Symptomatic patients with associated risk factors are more prone to increased morbidity and mortality. Rampant use of anti-fungals in asymptomatic patients have resulted in development of resistant candida strains. This study was aimed to identify the candida species in urinary specimens and determine their anti-fungal susceptibility pattern.

Materials and Methods: A total of 125 urinary samples received for mycological investigation were included in the study. Identification of candida till species level was done using routine methods. Antifungal susceptibility was carried out for fluconazole and amphotericin B using micro broth dilution technique.

Results: Candida albicans (44.2\%) was the commonest species which was isolated followed by Candida tropicalis (40.3\%). Predominately, isolates were obtained from ICU setup (49.3\%). Prolonged use of antibiotics was most common associated risk factor followed by catheterization. Candida isolates were more susceptible to amphotericin B than fluconazole.

Conclusions: Differentiation form colonization and species identification is important because of rise in more resistant non albicans candida. Association of candiduria with risk factors play an important role in defining the treatment protocol. Anti-fungal susceptibility testing have direct impact on patient care.

Keywords: Urinary tract infection, candida, antifungal susceptibility test.
\end{abstract}

\section{Introduction}

Candida is one of the commonest fungus causing human infections. The spectrum of infections ranges from mucocutaneous involvement to invasive fungal infections. Although Candida is a part of normal commensal flora colonizing skin, gastrointestinal tract and external genitalia, it plays an important role in urinary tract infections. ${ }^{[1]}$

Majority of urinary tract infections are caused by bacteria and only $10-15 \%$ are caused by fungi. Out of all the fungi, Candida species is most frequently isolated from urine. Candidal urinary tract infection may include cystitis, pyelonephritis 
which occurs due to retrograde infection specially in patients with urinary tract obstruction. Renal candidiasis may occur due to hematogenous seeding. ${ }^{[2]}$

Candiduria is defined as the presence of Candida in urine. It may include colonization (of urinary tract or indwelling catheter) or true infection of the urinary tract. True infection can be identified by presence of pyuria and by quantification of number of organisms in fresh urine sample. ${ }^{[2]}$

Among the Candida species, Candida albicans is the most frequently isolated species from urine samples. Non - albicans Candida like Candida glabrata, Candida tropicalis, Candida krusie, Candida parapsilosis isolation has been increased over the past two-three decades. ${ }^{[3]}$

Majority of patients with candiduria remain asymptomatic especially in catheterized patients not requiring any treatment. However, treatment is recommended for symptomatic patients as well as high risk patients like patients with urinary tract structural abnormality, patients undergoing urologic manipulations, neutropenia, infants with low birth weight, patients with renal allografts as these patients tend to have candidemia. According to IDSA guidelines, fluconazole or amphotericin $B$ is given for 7-14 days. ${ }^{[4]}$

Increased morbidity and mortality has been reported among invasive candidiasis patients with different degree of resistance to anti-fungal agents. In view of this, current study was undertaken to speciate and characterize Candida isolates from urine sample and their anti-fungal susceptibility testing was done against commonly used drugs, amphotericin B and fluconazole.

\section{Materials and Methods}

Study was conducted in the tertiary care hospital over a period of six months after obtaining ethics committee approval. During the study period, 125 urinary samples were received for mycological investigation. The specimens were received from the Intensive Care Units (ICU) as well as from other speciality units. Samples were processed as per standard protocol. Microscopy was done.
Specimen was inoculated on two Sabouraud Dextrose Agar (SDA) slants and were incubated at $30^{\circ} \mathrm{C}$ and $37^{\circ} \mathrm{C}$. Candida isolates were identified and speciated by term tube test, urease test, sugar fermentation test, sugar assimilation test and colour on CHROM agar candida medium. Dalmau plate culture on corn meal agar was done for microscopic morphology.

Anti-fungal susceptibility testing was done using broth microdilution method as per standard Clinical Laboratory Standards Institute (CLSI) guidelines, document M27-A3. ${ }^{[5]}$ Sterile, disposable, 96 well microdilution plates were used for susceptibility testing of fluconazole and amphotericin B. RPMI 1640 was used as the broth medium. The endpoint readings were taken. Endpoint for amphotericin B was defined as the minimum concentration inhibiting visual growth. For azoles, endpoint was the minimum concentration where there was a decrement of almost half of original turbidity. Minimum inhibitory concentration (MIC) values were determined at $48 \mathrm{hr}$ for the two anti-fungal agents used. Quality control was performed using $C$. parapsilosis ATCC 22019, C albicans ATCC90028 and C. krusei ATCC- 6258.

Clinical break points used for fluconazole were according to CLSI M27-S4 ${ }^{[6]}$ and for amphotericin, according to CLSI M27-A3 (Table No : 1)

\section{Results}

A total of 125 urine samples were obtained for fungal culture during the study period. Out of this, Candida was isolated from 77/125 (61.6\%) of urine samples. Female predominance was noted with 48/77 (62.3\%) female patients and 29/77 (37.7\%) male patients. Among these, majority of patients were in the age group more than 60 years (35.1\%) (Table No 2)

Most of the positive samples were from ICU (49.3\%), followed by urology ward (20.8\%), pediatric ward $(11.7 \%)$, surgery ward $(9.1 \%)$, Obs/gynae ward (5.2\%) and medicine ward (3.9\%) respectively. (Table No 3 ) 
Risk factors associated with candiduria were analyzed. Prolonged antibiotic therapy $(79.2 \%)$ was most common, followed by presence of indwelling urinary catheter $(75.3 \%)$. Diabetes mellitus $(15.6 \%)$, pregnancy $(7.8 \%)$ and urinary tract abnormality (5.2\%) like obstruction and neurogenic bladder were other associated risk factors.

Among 77 cases of candiduria, 34/77 (44.2\%) were due to $C$. albicans and 43/77 (55.8\%) were due to non- albicans Candida. Among nonalbicans Candida, C. tropicalis (40.2\%) was most commonly isolated followed by, $C$. glabrata (7.8\%), C. kefyr (2.6\%), C. parapsilosis (2.6\%), and $C$. lusitaniae (2.6\%). (Figure No: 1)
A total of $25(73.5 \%)$ C. albicans were sensitive and $3(8.8 \%)$ were susceptible dose dependent to fluconazole, giving overall sensitivity of $82.3 \%$ to fluconazole. Six $(17.6 \%)$ isolates of $C$. albicans were resistant to fluconazole. (Table No: 4)

Among non- albicans Candida, overall sensitivity to fluconazole is $67.4 \%$ (29/43). Candida tropicalis and $C$. glabrata were major nonalbicans Candida which were resistant to fluconazole.

Susceptibility to amphotericin B was $92.2 \%$ among all candida species, of which $C$. albicans accounted for $94.1 \%$ and non- albicans Candida $90.7 \%$ respectively.

Table No 1: MIC value interpretation for anti-fungals

\begin{tabular}{|l|c|c|c|}
\hline & Susceptible (S) & $\begin{array}{c}\text { Susceptible Dose Dependent } \\
\text { (SDD) }\end{array}$ & Resistant (R) \\
\hline Amphotericin B & $\leq 1 \mu \mathrm{g} / \mathrm{ml}$ & - & $\geq 2 \mu \mathrm{g} / \mathrm{ml}$ \\
\hline Fluconazole & $\leq 2 \mu \mathrm{g} / \mathrm{ml}$ & $2-8 \mu \mathrm{g} / \mathrm{ml}$ & $\geq 8 \mu \mathrm{g} / \mathrm{ml}$ \\
\hline
\end{tabular}

Table No 2: Age and Gender distribution of patients

\begin{tabular}{|l|c|c|c|}
\hline Age Group & Male & Female & Total \\
\hline$<15$ & 7 & 9 & $16(20.8 \%)$ \\
\hline $16-30$ & 3 & 11 & $14(18.2 \%)$ \\
\hline $31-45$ & 2 & 6 & $8(10.4 \%)$ \\
\hline $46-60$ & 4 & 8 & $12(15.6 \%)$ \\
\hline$>60$ & 13 & 14 & $27(35.1 \%)$ \\
\hline Total & $29(37.7 \%)$ & $48(62.3 \%)$ & 77 \\
\hline
\end{tabular}

\begin{tabular}{|l|c|c|}
\hline \multicolumn{3}{|c|}{ Table No 3: Isolation of Candida from different units } \\
\hline & TOTAL & PERCENTAGE (\%) \\
\hline ICU & 38 & 49.3 \\
\hline Urology ward & 16 & 20.8 \\
\hline Pediatric ward & 9 & 11.7 \\
\hline Surgery ward & 7 & 9.1 \\
\hline Obs/gynae ward & 4 & 5.2 \\
\hline Medicine ward & 3 & 3.9 \\
\hline
\end{tabular}


Table No 4: Anti-fungal susceptibility of candida spp to Fluconazole and Amphotericin B

\begin{tabular}{|l|c|c|c|c|c|}
\hline \multirow{2}{*}{$\begin{array}{l}\text { Candida Species } \\
(n)\end{array}$} & \multicolumn{3}{|c|}{ Fluconazole } & \multicolumn{2}{c|}{ Amphotericin B } \\
\cline { 2 - 5 } & $\mathbf{S}(\leq 2 \mu \mathrm{g} / \mathrm{ml})$ & $\begin{array}{c}\text { SDD } \\
(2-8 \mu \mathrm{g} / \mathrm{ml})\end{array}$ & $\mathbf{R}(\geq 8 \mu \mathrm{g} / \mathrm{ml})$ & $\mathbf{S}(\leq 1 \mu \mathrm{g} / \mathrm{ml})$ & $\mathbf{R}(\geq 2 \mu \mathrm{g} / \mathrm{ml})$ \\
\hline C. albicans $(\mathrm{n}=34)$ & 25 & 3 & 6 & 32 & 2 \\
\hline C. tropicalis (n=31) & 22 & 2 & 7 & 29 & 2 \\
\hline C. glabrata (n=6) & 1 & 1 & 4 & 5 & 1 \\
\hline C. kefyr (n=2) & 0 & 1 & 1 & 1 & 1 \\
\hline C. parapsilosis (n=2) & 0 & 0 & 2 & 2 & 0 \\
\hline C. lusitanae (n=2) & 2 & 0 & 0 & 2 & 0 \\
\hline
\end{tabular}

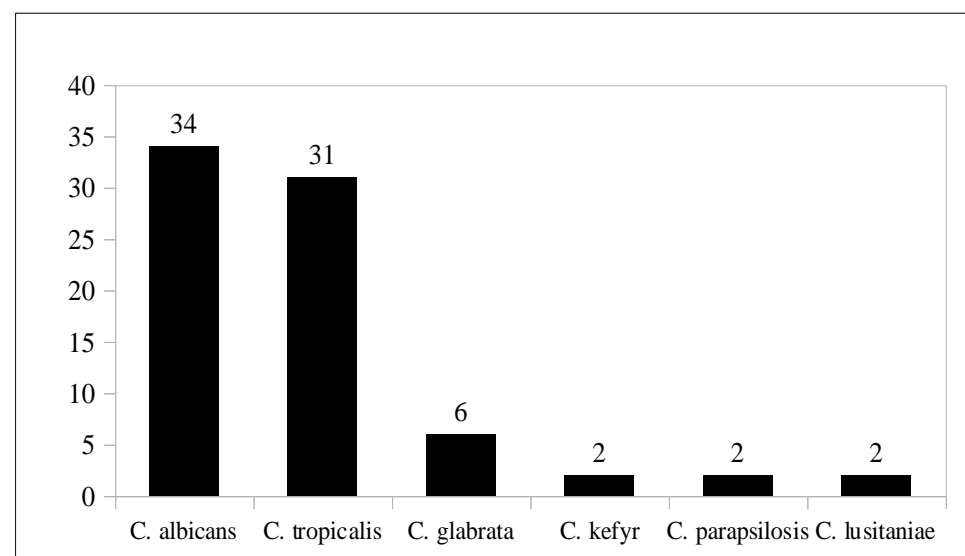

Figure 1 : Species distribution of Candida isolates

\section{Discussion}

Urinary tract infections by candida is increasing worldwide specially in hospitalized patients. Candida spp colonization is seen in $5-15 \%$ of hospitalized patients. This rate increases drastically up to 50 to $80 \%$ when patients are admitted in ICU. ${ }^{[3,7]}$

Extremes of age, renal transplants, HIV infection, diabetes, prolonged antibiotic therapy are associated with increased candidal colonization. When there is a breach in body defenses as seen in instrumentation including catheterization, urologic procedures, immunosuppressive therapy, candidal colonization is aggravated and may sometimes lead to candidemia. ${ }^{[8]}$

In our study, majority of patients $(35.1 \%)$ belong to age group above 60 years which was similar to other studies. ${ }^{[9]}$ This may be attributed to the fact that as age advances, the immunity starts waning. In addition, frequent hospitalization and catheterization owing to the prostatic ailments are common in elderly population. ${ }^{[10]}$

The incidence of candiduria was found to be greater in females $(62.3 \%)$ when compared with males. This affiliation towards female sex may be due the vaginal colonization by candida in healthy women or due to vaginal candidiasis. These yeasts then ascend urethra thus causing urinary tract infections. [11]

Analyzing other possible risk factors contributing to the infection, we found that $79.2 \%$ had history of prior use of broad spectrum antibiotics, $75.3 \%$ were catheterized whereas $15.6 \%$ had diabetes.

Long term use of antibiotics suppress the endogenous bacterial flora leading to increased colonization of gut and genital tract by Candida spp. This may inadvertently lead to candiduria especially in presence of an indwelling catheter which itself facilitates yeast cell adhesion. ${ }^{[12]}$ 
Also, greater incidence of candiduria in diabetic patients can be explained by the fact that acidic urine provides favorable environment for growth of Candida yeast cells, facilitating colonization of bladder. ${ }^{[8]}$

Intensive care unit admission exposes patient to many such risk factors. This increases incidence of candidal infections in these critical patient units. This fact is corroborated by previous studies. ${ }^{[3,13]}$ In our study, we also found higher incidence $(49.3 \%)$ of candiduria from intensive care units.

There has been an epidemiological shift with emergence of non- albicans Candida as predominant species causing urinary tract infection. ${ }^{[14]}$ In our study, non- albicans Candida constituted $66.7 \%$ of isolates with $C$. tropicalis being most common non- albicans Candida. Nonalbicans Candida are not only well adapted to the urinary tract environment but also are more resistant to anti-fungal drugs rendering their eradication difficult. ${ }^{[3]}$

In our study, susceptibility to fluconazole was found to be lesser for non- albicans Candida (67.4\%) when compared with $C$. albicans (82.3\%). Candida isolates were more susceptible to amphotericin B $(92.2 \%)$ than fluconazole (74\%). This finding is substantiated by Yashvanth et al. ${ }^{[15]}$ Fluconazole resistance was seen in $17.6 \%$ of $C$. albicans and $22.6 \%$ of C. tropicalis, corelating with other studies. ${ }^{[16]}$

Among all the candida, C. albicans, in general is more sensitive to common anti-fungal agents. However azole resistant $C$. albicans have been reported in HIV infected patients. ${ }^{[17]}$

Susceptibility for azoles vary with different nonalbicans Candida. Candida tropicalis and $C$. parapsilosis are regarded as most susceptible to azoles. Various mechanisms of resistance at molecular level are studied. Altered plasma membrane and increased active drug efflux play key role in development of multi-drug resistance in C. tropicalis and C. glabrata respectively. ${ }^{[18,19]}$ Apart from being intrinsically resistant to azoles, C. glabrata, also show reduced susceptibility to amphotericin B. In such cases, high dose of amphotericin is required especially in immunocompromised patients. ${ }^{[4]}$ Candida glabrata isolated in our study have shown resistance to flucanozole and amphotericin B.

Anti-fungal treatment is not recommended in patients with asymptomatic candiduria as even simple removal of catheter may help. Treating such patients overtly even single prophylactic dose can lead to fluconazole resistance. This might be due to the selective pressure due to its fungistatic nature. Azole drugs used in these asymptomatic patients usually suppresses sensitive flora with replacement by more resistant strains. ${ }^{[18,20]}$

Fluconazole resistant Candida isolates usually show cross-resistant to other azoles and even to polyenes as well. Thus, resistance to amphotericin B may develop in such patients. This crossresistance is because of many molecular mechanisms especially due to over-expression of CDR genes. ${ }^{[20]}$

Critically ill patients with associated risk factors develops candidemia with high morbidity and mortality. In such scenario, early diagnosis with species identification and anti-fungal susceptibility testing is mandate for initiation of adequate therapy, as many Candida species are developing resistance.

\section{Conclusion}

Distinction of colonization with the true infection is important to avoid overzealous treatment by anti fungal agents leading to emergence of resistance. Species identification, individualized anti-fungal susceptibility pattern and risk stratification are mainstay for treatment of urinary candidal infection. Development of high-level azole resistance is a matter of concern.

Acknowledgements: NA

Sources of support in the form of grants: NIL 


\section{References}

1. Achkar JM, Fries BC. Candida infections of the genitourinary tract. Clinical microbiology reviews. 2010 Apr 1;23(2):253-73.

2. Kauffman CA. Candiduria. Clinical Infectious Diseases. 2005 Sep 15;41(Supplement_6):S371-6.

3. Sobel JD, Fisher JF, Kauffman CA, Newman CA. Candida urinary tract infections epidemiology. Clinical infectious diseases. 2011 May 15;52(suppl_6):S433-6.

4. Pappas PG, Kauffman CA, Andes DR, Clancy CJ, Marr KA, Ostrosky-Zeichner L, Reboli AC, Schuster MG, Vazquez JA, Walsh TJ, Zaoutis TE. Clinical practice guideline for the management of candidiasis: 2016 update by the Infectious Diseases Society of America. Clinical Infectious Diseases. 2015 Dec 16;62(4):e1-50.

5. Clinical Laboratory Standards Institute, CLSI Document M27- A3: Reference Method for Broth Dilution Antifungal Susceptibility Testing of Yeasts, Approved Standard, CLSI, Wayne, PA, USA, 2008a.

6. Clinical Laboratory Standards Institute, CLSI document M27- S4: Reference Method for Broth Dilution Antifungal Susceptibility Testing of Yeasts, Approved Standard, CLSI, Wayne, PA, USA, 2012.

7. Eggimann $\mathrm{P}$, Garbino J, Pittet D. Epidemiology of Candida species infections in critically ill nonimmunosuppressed patients. The Lancet infectious diseases. 2003 Nov 1;3(11):685702.

8. Fisher JF, Kavanagh K, Sobel JD, Kauffman CA, Newman CA. Candida urinary tract infection: pathogenesis. Clinical Infectious Diseases. 2011 May 15;52(suppl_6):S437-51.

9. Goyal RK, Sami H, Mishra V, Bareja R, Behara RN. Non-albicans candiduria: an emerging threat. J Appl Pharm Sci. 2016 Mar;6(3):48-50.

10. Jacobs LG. Fungal urinary tract infections in the elderly. Drugs \& aging. 1996 Feb 1;8(2):89-96.

11. Silva V, Hermosilla G, Abarca C. Nosocomial candiduria in women undergoing urinary catheterization. Clonal relationship between $\mathrm{s}$ trains isolated from vaginal tract and urine. Medical mycology. 2007 Nov 1;45(7):645-51.

12. Fisher JF, Chew WH, Shadomy S, Duma RJ, Mayhan CG, House WC. Urinary tract infections due to Candida albicans. Reviews of infectious diseases. 1982 Nov 1;4(6):1107-18.

13. Aubron C, Suzuki S, Glassford NJ, GarciaAlvarez M, Howden BP, Bellomo R. The epidemiology of bacteriuria and candiduria in critically ill patients. Epidemiology \& Infection. 2015 Feb;143(3):653-62.

14. Goel R, Singh S, Gill AK, Kaur A, Kour I. Speciation, Characterization and Antifungal Susceptibility Pattern of Candida Species. Journal of Contemporary Medical Research. 2018;5(5):E1-4.

15. Yashavanth R, Shiju M.P, Bhaskar U.A, Ronald R, Anita K.B. Candiduria: Prevalence and Trends in Antifungal Susceptibility in A Tertiary Care Hospital of Mangalore. Journal of Clinical and Diagnostic Research, 2013; 7(11): 24592461.

16. Singla N, Gulati N, Kaistha N, Chander J. Candida colonization in urine samples of ICU patients: determination of etiology, antifungal susceptibility testing and evaluation of associated risk factors. Mycopathologia. 2012 Aug 1;174(2):14955.

17. Kelly, S. L., D. C. Lamb, D. E. Kelly, J. Loeffler, and H. Einsele. 1996. Resistance to fluconazole and amphotericin in Candida albicans from AIDS patients. Lancet 348:1523-1524. 
18. Kołaczkowska A, Kołaczkowski M. Drug resistance mechanisms and their regulation in non-albicans Candida species. Journal of Antimicrobial Chemotherapy. 2016 Jan 21;71(6):1438-50.

19. Kanafani ZA, Perfect JR. Resistance to antifungal agents: mechanisms and clinical impact. Clinical Infectious Diseases. 2008 Jan 1;46(1):120-8.

20. White TC, Marr KA, Bowden RA. Clinical, cellular, and molecular factors that contribute to antifungal drug resistance. Clinical microbiology reviews. 1998 Apr 1;11(2):382-402. 